\title{
Corrigendum to “In Vivo Quantitative Ultrasound Image Analysis of Femoral Subchondral Bone in Knee Osteoarthritis"
}

\author{
Jana Podlipská, ${ }^{1}$ Juhani M. Koski, ${ }^{2}$ Pasi Pulkkinen, ${ }^{1}$ and Simo Saarakkala ${ }^{1,3,4}$ \\ ${ }^{1}$ Research Unit of Medical Imaging, Physics and Technology, University of Oulu, Aapistie 7A, POB 5000, 90014 Oulu, Finland \\ ${ }^{2}$ Department of Internal Medicine, Mikkeli Central Hospital, 50100 Mikkeli, Finland \\ ${ }^{3}$ Department of Diagnostic Radiology, Oulu University Hospital, 90029 Oulu, Finland \\ ${ }^{4}$ Medical Research Center, University of Oulu and Oulu University Hospital, 90014 Oulu, Finland
}

Correspondence should be addressed to Jana Podlipská; jana.podlipska@oulu.fi

Received 28 April 2016; Accepted 26 July 2016

Copyright (C) 2016 Jana Podlipská et al. This is an open access article distributed under the Creative Commons Attribution License, which permits unrestricted use, distribution, and reproduction in any medium, provided the original work is properly cited.

In the article titled "In vivo quantitative ultrasound image analysis of femoral subchondral bone in knee osteoarthritis" [1], there was an error in the data set (scatter plot) of provided Figure 2(d). The correlation, however, was correct and consequently it does not change our conclusions. Here we provide Figure 2(d) with the corrected data set. Additionally, in Figure 2(a) the mathematical expression of $P$ value was corrected from " $P=0.000$ " to more appropriate " $P<0.001$."

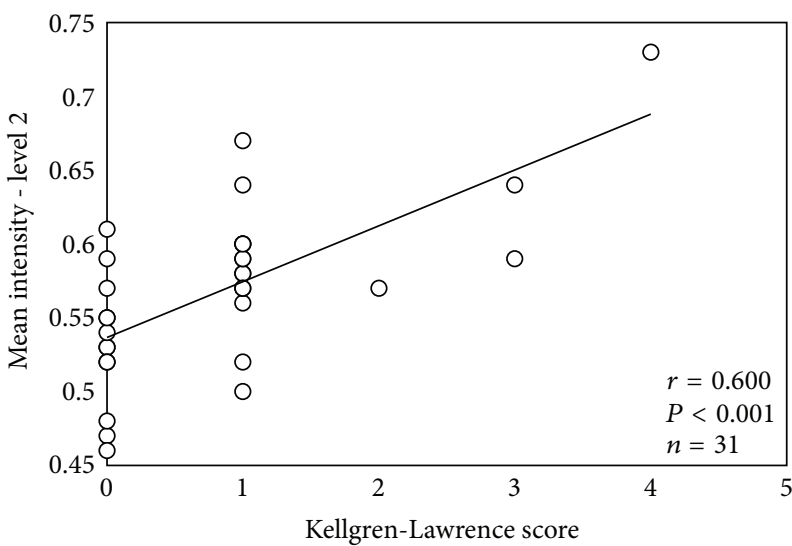

(a)

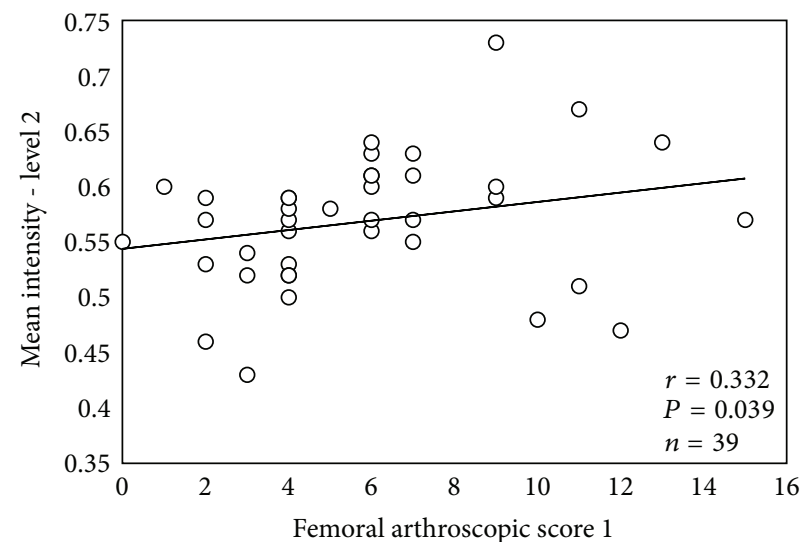

(b)

Figure 2: Continued. 


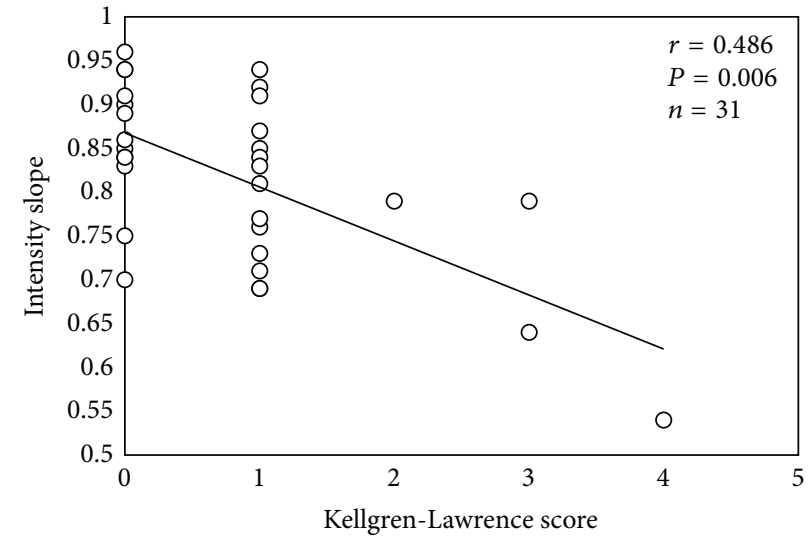

(c)

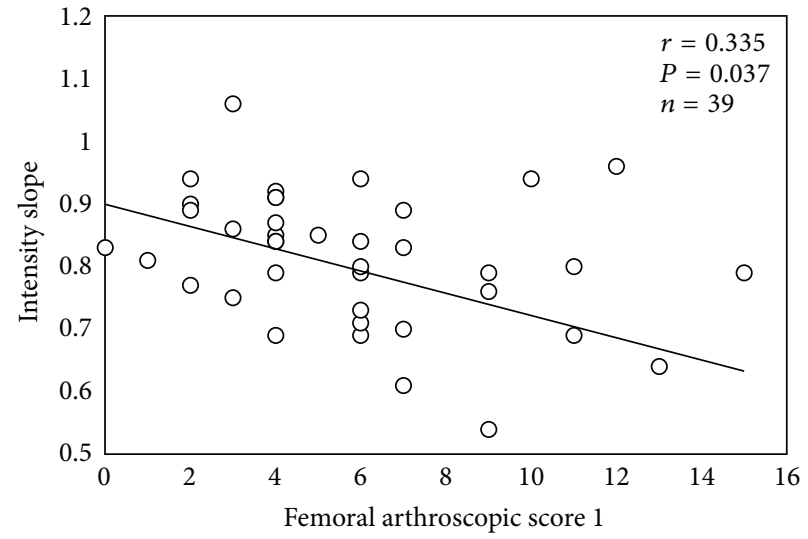

(d)

FIGURE 2: (a) Relationship between normalized mean intensity values of femoral bone level 2 and Kellgren-Lawrence (K-L) grading or femoral arthroscopic score 1 (FAS1) (b). Relationship between femoral subchondral bone intensity slope and K-L grading (c) or FAS1 (d). The slope was calculated from the first 2 levels. Please note that the trendline in each plot is only for illustration purposes.

\section{References}

[1] J. Podlipská, J. M. Koski, P. Pulkkinen, and S. Saarakkala, "In vivo quantitative ultrasound image analysis of femoral subchondral bone in knee osteoarthritis," The Scientific World Journal, vol. 2013, Article ID 182562, 8 pages, 2013. 


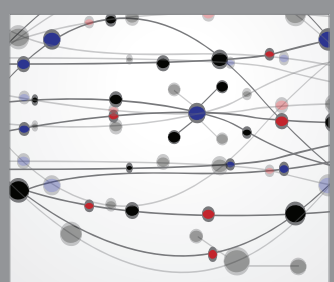

The Scientific World Journal
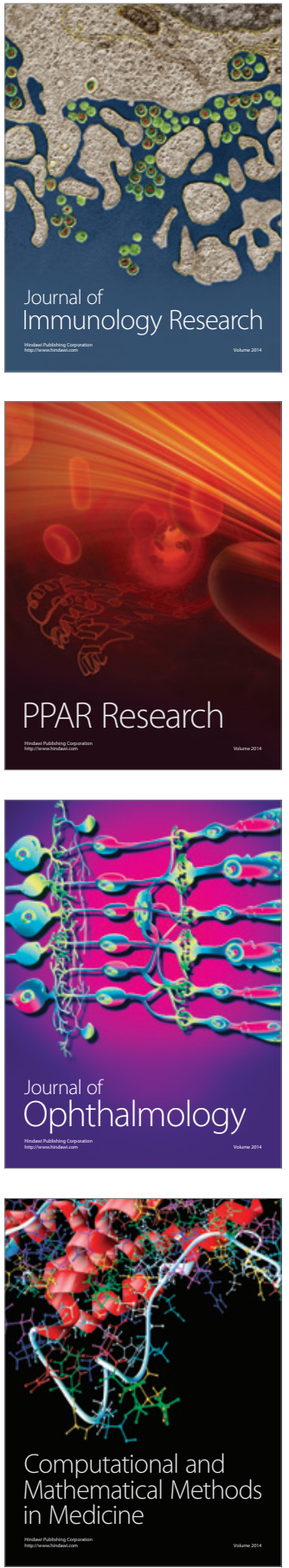

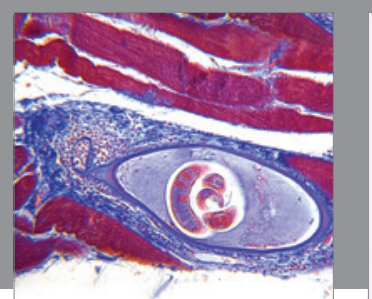

Gastroenterology Research and Practice

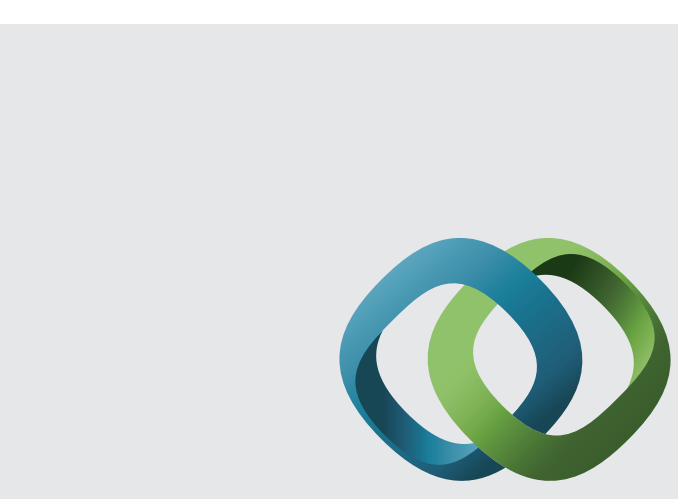

\section{Hindawi}

Submit your manuscripts at

http://www.hindawi.com
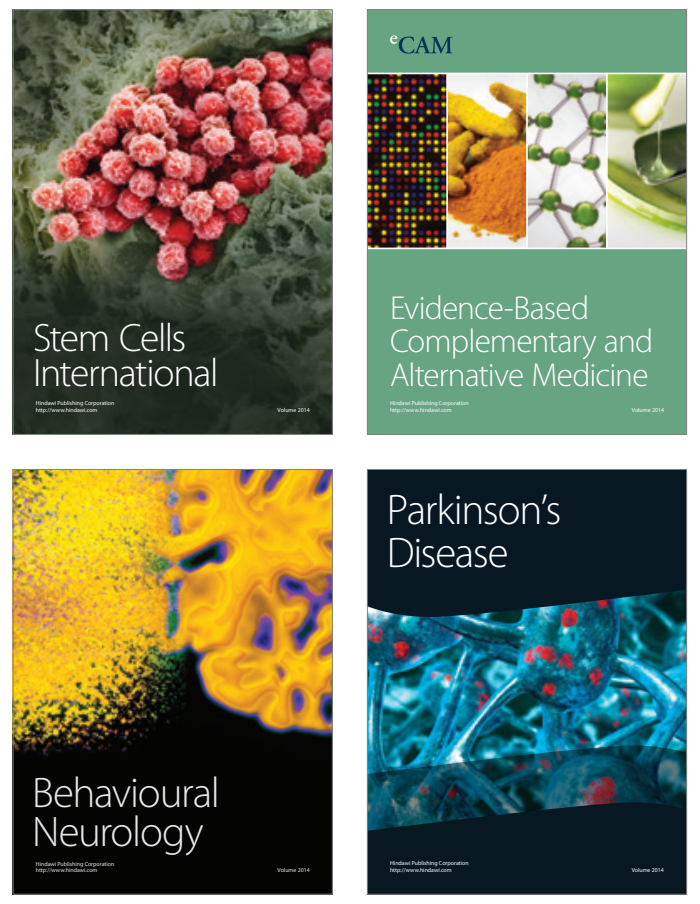
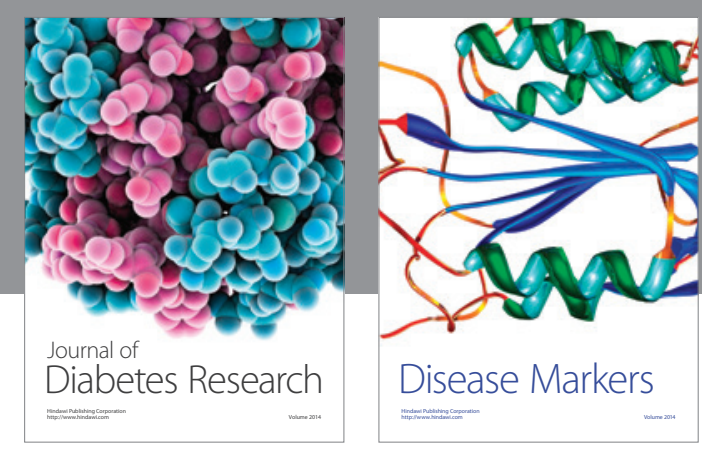

Disease Markers
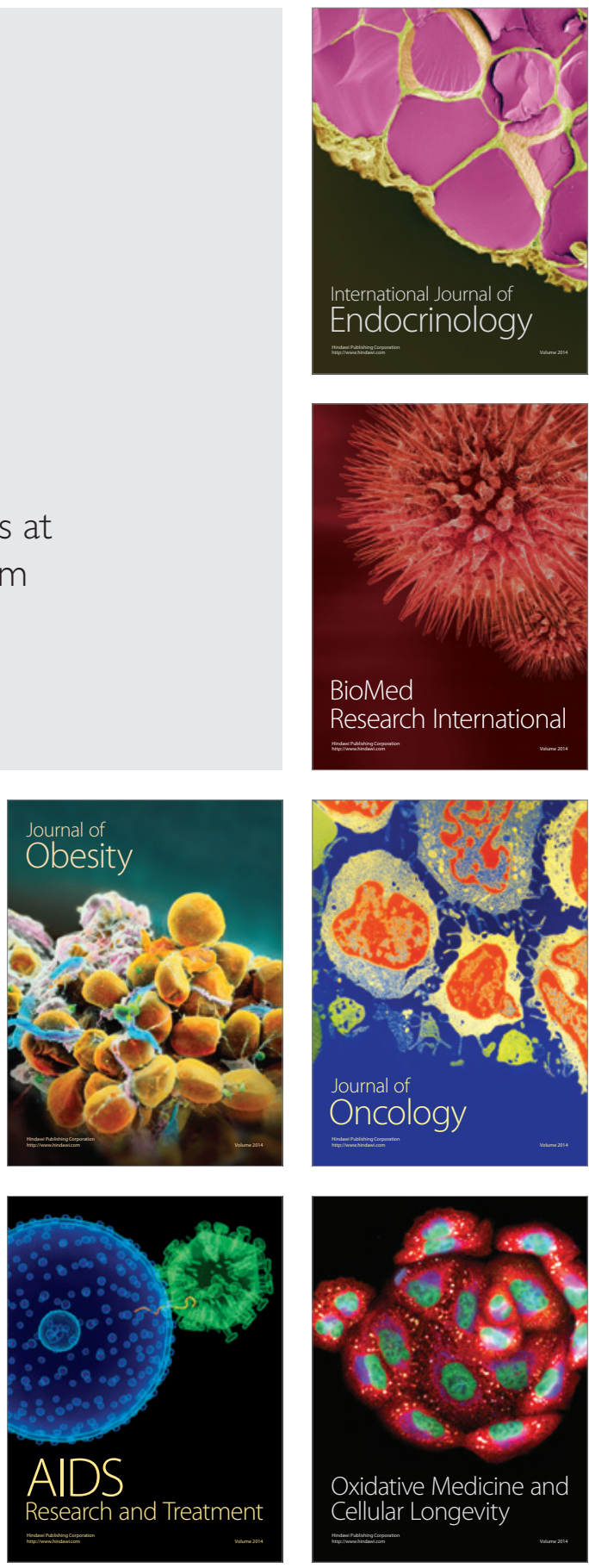